\title{
Cholera (Blue Death Disease)
}

\author{
Rashida Qari ${ }^{1 *}$ and Rasikha Naseem ${ }^{2}$ \\ ${ }^{1}$ Maritime Studies Department, Faculty of Humanities and Social Sciences, Bahria University Karachi Campus, Pakistan \\ ${ }^{2}$ Department of Pharmaceutics, Faculty of Pharmacy, University of Karachi, Pakistan \\ *Corresponding Author: Rashida Qari, Maritime Studies Department, Faculty of Humanities and Social Sciences, Bahria University \\ Karachi Campus, Pakistan.
}

Received: June 14, 2019; Published: July 03, 2019

DOI: $10.31080 /$ ASMI.2019.02.0293

Cholera also known by the name of 'blue death', is an infectious disease still prevalent in some of the countries majorly towards the developing countries. Cholera is caused by micro bacteria 'Vibrio Cholera'. It is a deadly disease due to the damage of human body by dehydrating through excretion of large amounts of body fluids and skin become bluish green. Dehydration is so severe that at times person suffering through dies. It has been an epidemic outbreak for many years and according to reports of 2010, 3 million to $5 \mathrm{mi}$ llion people get cholera every year and according to one estimate of World Health Organization (WHO) 58,000-150,000 people die from the cholera disease every year. It is still a pandemic and is considered deadly for children. In October 2018 WHO reported that in all suspected cases approximately $30 \%$ are children about five years old.

Recently in the Celebes Islands Indonesia seventh pandemic, started and globally spread too approximately in above hundred countries [1]. Cholera is mainly caused by ingestion of food or water that is unclean and it is thought to be contaminated with feces of a person infected with the mycobacterium. It is considered to spread due to poor sanitary conditions. The disease is although not spread from person to person by contact. When the contaminated food or water reaches the intestines of a person, a toxin is released that causes severe watery diarrhea along with vomiting which is responsible for the elimination of heavy amounts of fluids from the body which causes excessive dehydration. The Oral Rehydaration Salt solution (ORS) is an old and most effective solution to control the effect of cholera, used globally for save life affected by cholera.

There is significant relationship is found in between cholera disease and climate change that is human health intricately link to the seasons, local weather pattern and other environmental factors
[2]. The symptoms appear suddenly within one to five days which is known as cholera's incubation period. The treatment should begin as soon the symptoms appear though there is no proper cure but intravenous or oral hydration of important electrolytes such as potassium and sodium and fluid should be carried out to prevent dehydration. If person infected with vibrio cholera is given proper treatment, he can be saved from death due to excessive dehydration. Only some of the strains of vibrio cholera are considered more dangerous than others to an extent that a person infected with these strains have only two hours to save their life.

A vaccine is available in oral form against cholera which provides protection for six months. Cholera can only be prevented by taking safety measures to enhance the quality of hygiene, some measures considered worldwide safe against cholera are washing hands after using toilet, washing hands with a soap before eating food, thoroughly cooked food, use of fresh fruits and vegetables etc. Water for drinking should boiled or minerals or treated water with chlorine and iodine, even tea or coffee should made within above mention waters to eliminate the strain of infectious vibrio cholera from the water.

In developed countries, cholera is not a threat anymore due to high quality of sanitary conditions and proper sewage systems which help in treating the wastes before being drained into the environment. Governments and world health organizations can take proper steps to eliminate the risk of cholera from developing and under developed countries by prompting the safe measures as per suggestions from the agencies like World Health Organization and Centre for the Disease control and Prevention.

Cholera is very dangerous disease, can kill within hours if untreated. According to recent news phage therapy is being used for 
the protection of human from cholera disease. Phage therapy or viral phage therapy is the therapeutic use of bacteriophages to treat pathogenic infections and it is very important in human medicine beside the veterinary and agriculture but this therapy is very costly. WHO Collaborating Centre for phage typing and drug evaluated the phage typing method. Research antiplaque Institute has also developed Phage therapy scheme and utilizes seven virulent phages of stable activity.

The bacteria "Vibrio Cholera" survive well in any water. In the sea remain viable in most of the plants and animals such as crustaceans (copepods), shellfish, algal plants and plankton both phytoplankton and zooplanktons. The bacteria $V$. cholera can survive about fifty days in feces. Cholera is deadly disease that's why still research on different aspect of cholera is continue to decrease the percentage of suffering people affected by cholera. Many algal plants are used for anticholera disease. Alga-produced cholera toxin-Pfs 25 fusion proteins as oral vaccines. It is low-cost vaccine for those countries which are poor and developing. The interesting thing is that it is orally delivered, no expensive purification and no injectable delivery. Gregory., et al. [3] reported that the chloroplast of the eukaryotic alga Chlamydomonas reinhardtii to produce a chimeric protein consisting of the 25-kDa Plasmodium falciparum surface protein (Pfs25) fused to the $\beta$ subunit of the cholera toxin (CtxB) to investigate an alga-based whole-cell oral vaccine.

\section{Bibliography}

1. Hu D., et al. "Origins of the current seventh cholera pandemic". PNAS (2016): E7730-E7739|.

2. Lipp EL., et al. "Effects of Global Climate on Infectious Disease: the Cholera Model”. Clinical Microbiology Reviews 15.4 (2000): 757-770.

3. Gregory JA., et al. "Alga-Produced Cholera Toxin-Pfs25 Fusion Proteins as Oral Vaccines". Applied and Environmental Microbiology 79.13 (2013): 3917-3925.

Volume 2 Issue 8 August 2019

(C) All rights are reserved by Rashida Qari and Rasikha Naseem. 